\title{
EL EPISODIO CRÍTICO COMO UNA HERRAMIENTA DIDÁCTICA PARA SIGNIFICAR LOS APORTES DE DIFE- RENTES TEORÍAS EN LA FORMACIÓN CONTINUA DE PROFESORES
}

\section{THE CRITICAL EPISODE AS A DIDACTIC TOOL TO SIGNIFY THE CONTRIBUTIONS OF THE DIFFERENT THEORIES ON THE CONTINUOUS TRAINING OF TEACHERS}

\author{
Eduardo Carrasco Henríquez¹, Teresa Sofía Oviedo Millones²
}

\section{RESUMEN}

Diversas perspectivas teóricas ofrecen herramientas para mejorar las prácticas docentes. Sin embargo se muestra, en diversas investigaciones, que los docentes construyen su práctica desde sus vivencias con los estudiantes. En este artículo se reporta una experiencia de formación continua a docentes de maestría, que considera central el relato protagónico de situaciones críticas vividas en la práctica docente para fortalecer competencias de análisis crítico. El relato protagónico se estructura en tres facetas: (a) la descripción externa de la situación desde la posición de un observador objetivo; (b) la descripción del devenir emocional del protagonista que concurrió en la situación vivida y (c) un relato de la racionalidad pedagógica, en el que se tomaron decisiones didácticas. A partir de ello, los profesores analizan, desde diferentes teorías, la situación y elaboran alternativas de acción didáctica. La metodología es un estudio de caso, en el marco de investigación protagónica. El análisis de datos se basa en el análisis conceptual de las descripciones de los docentes en formación. Los resultados de la actividad muestran una mayor valoración positiva de la producción científica en didáctica de las matemáticas como herramientas para un mejor análisis de lo vivido, construyendo alternativas de acción contextuadas a su práctica profesional.

PALABRAS CLAVE: Formación de profesores. Experiencias docentes. Análisis de la propia práctica

\section{ABSTRACT}

Different theoretical perspectives offer tools to improve teaching practices. But, the research show that teachers build practice from their students experience. In this article we report an experience of continuous training. The teacher develop critical analysis skill from one protagonist story of critical teaching situations. The report is structure in three moments: (a) The external description of the situation. It is a report of a objective observer; (b) The description of the teacher emotions that concurred in the lived situation and; (c) a description of the pedagogical rationality, in which didactic decisions were taken. The next step teachers analyze critical situation using different theories for propose didáctical alternatives. The report is a case study, in the framework of protagonist investigation. The data analysis is made by a conceptual analysis of the descriptions of the teachers. The results of the activity show a greater positive evaluation. Teacher develop skills to use the products of mathematic education as tools of didactical analysis of their experience.

KEYWORDS: Teacher training. Teaching experiences. Analysis of the practice itself.

\footnotetext{
${ }_{1}^{1}$ Dr. en Matemática Educativa, Investigador de la Universidad Metropolitana de Ciencias de la Educación. Departamento de Educación Básica, área de Matemática. Coordinador de Investigación. eduardo.carrasco@umce.cl

${ }^{2}$ Dra(c). en Educación Matemática, Pontificia Universidad Católica del Perú. Miembro del Grupo de Investigación EDUTEC (PUCP).sofia.oviedo@pucp.edu.pe.
} 


\section{INTRODUCCIÓN}

Un aspecto central en la investigación sobre la formación de profesores refiere al saber docente y la inadecuada comprensión de la naturaleza teórico -práctica de la pedagogía (LABRA, 2011). Esto implica una dificultad para abordar procesos de mejora de la propia práctica.

Las diversas perspectivas teóricas ofrecen herramientas para mejorar las prácticas docentes. Sin embargo diversas investigaciones muestran que los docentes construyen su práctica desde sus vivencias con los estudiantes, por tanto los docentes requieren desarrollar procesos reflexivos más críticos sobre la práctica pedagógica que impacten efectivamente en los resultados de aprendizaje del estudiantado (HERNÁNDEZ Y CATRINAO, 2016). Esto plantea el desafío de fortalecer en la formación de profesores estrategias que promuevan competencias para el análisis crítico de la práctica desde elementos teóricos de didáctica de las matemáticas.

Es reconocido que los profesores tienen la capacidad de actuar para el logro de metas, para la toma de decisiones y elecciones, por ejemplo, en relación con su trabajo e identidad profesional (ETELÄPELTO, VÄHÄSANTANEN, HÖKKÄ, Y PALONIEMI, 2013; KETELAAR, BEIJAARD, BOSHUIZEN, Y DEN BROK, 2012; LASKY, 2005; LIPPONEN Y KUMPULAINEN, 2011). Por lo cual en los esfuerzos de cambio, las visiones profesionales personales son un factor relevante para determinar cuándo y cómo los profesores - y otros miembros clave de la comunidad escolar - realizan esfuerzos para el cambio (HAMMERNESS, 2010). Es decir, uno de los aspectos clave para un proceso de cambio educativo es considerar la visión de los profesores y para ello se hace central el desarrollo de competencias para el análisis crítico de la propia práctica en la formación docente.

En particular, y en la perspectiva de un cambio que apunte al mejoramiento de la calidad de la educación, es fundamental considerar la transformación de la relación pedagógica cotidiana. Es decir, a partir de los problemas cotidianos que viven los profesores incorporar, como elemento central de cambio, la modificación de la práctica docente. Luego un real proceso de cambio requiere, entonces, de la participación activa de sus profesores (ASSAÉL, GUZMAN,
1992, REYES, CORNEJO, ARÉVALO Y SÁNCHEZ, 2010). Así en la reflexión colectiva se potencian aspectos para una mirada crítica que no solo aborda los obstáculos de los estudiantes, o de la institución en que se ejerce, sino también de la propia práctica y como ella posibilita el aprendizaje.

En este marco, este artículo reporta una experiencia de formación continua, en el marco de una maestría profesional de enseñanza de la matemática, que considera central el análisis de situaciones críticas vividas por los profesores. A partir de un relato protagónico de situaciones en que el docente reconoce una solución poco efectiva (en la que la respuesta que da a sus alumnos no queda clara para ellos y en consecuencia, tampoco le satisface al docente), y desde el análisis compartido y colectivo construir propuestas alternativas de enseñanza

\section{ELEMENTOS TEÓRICOS}

El pensamiento crítico se concibe como el pensamiento racional y reflexivo interesado en decidir qué hacer o creer. Es decir, por un lado, constituye un proceso cognitivo complejo de pensamiento que reconoce el predominio de la razón sobre las otras dimensiones del pensamiento. Su finalidad es reconocer aquello que es justo y aquello que es verdadero, es decir, el pensamiento de un ser humano racional (ENNIS 1985, citado en LÓPEZ, 2013, p. 43). Nieto y Saiz (2011) mencionan que lo fundamental para desarrollar el pensamiento crítico son las competencias metacognitivas y la evaluación epistemológica (pensar sobre lo que se piensa). López (2013, p. 44) manifiesta que el pensamiento crítico

Es el tipo de pensamiento que se caracteriza por manejar, dominar las ideas. Su principal función no es generar ideas sino revisarlas, evaluarlas y repasar qué es lo que se entiende, se procesa y se comunica mediante los otros tipos de pensamiento (verbal, matemático, lógico, etcétera.

El taller, en la que los docentes realizaron el análisis de situaciones vividas en el aula con sus alumnos, involucra, como herramientas para el análisis crítico de la práctica, elementos de un relato protagónico desarrollado en los años noventa como una estrate- 
gia de perfeccionamiento docente en Chile (ASSAÉL; GUZMAN, 1992). Este relato se orienta a una mirada crítica en la medida que se preguntan por la historicidad y contextualización de las prácticas educativas que son parte de la práctica social. Son indagaciones docentes que siguen un método determinado que, más que aportar saber científico buscan construir mejores alternativas de acción pedagógica y que potencian una mirada rigurosa y que elabora nuevas comprensiones acerca de los problemas que analizan. El relato protagónico, como un medio privilegiado para acceder a la experiencia pedagógica vivida en la propia práctica, se constituye en un relato en primera persona, que permite entender cómo concurre lo acaecido, con la vivencia emocional y las representaciones pedagógicas que están a la base del actuar docente. Es una relato propio, que en la indagación colectivo va emergiendo un relato en segunda persona (en el sentido de Varela, 2000) de la situación que permite objetivar entre el colectivo aspectos centrales de la situación y de este modo dejar una relato para la indagación matemático educativa.

Para el análisis se optan por elementos de tres teorías diferentes para mirar la actividad de enseñanza matemática de los profesores:

\section{REGISTROS DE REPRESENTACIÓN SEMIÓTICA.}

Duval (2004) asume que la construcción del conocimiento matemático sólo es posible en la medida que se construyen sus diversas representaciones semióticas. Duval (2004) señala central el rol que las representaciones semióticas tienen en la actividad matemática, un rol que va más allá de la comunicación y que es parte constituyente de la construcción de saber matemático, es decir, "la semiosis es la que determina las condiciones de posibilidad y de ejercicio de la noesis" (DUVAL, 2004, p. 16). Esta actividad semiótica se constituye en tres tipos de acción: (a) la formación de representaciones en un registro, que implica la selección de signos apropiados dentro del conjunto de signos utilizados en dicho registro y la posterior combinación de acuerdo a las reglas de conformidad; (b), El tratamiento (transformación efectuada dentro de un mismo registro) y; (c) la conversión, que son transformaciones externas al registro de partida y con frecuencia no hay reglas que sistematizan o regulan su ejecución. Los tratamientos y las conversiones son actividades cognitivas ligadas a la posibilidad de transformación de las representaciones semióticas. (DISTÉFANO, AZNAR Y POCHULU, 2016).

\section{PRAXEOLOGÍA MATEMÁTICA}

Chevallard (2007) señala que una Organización Matemática, propuesta en un proceso de enseñanza, se compone de un bloque práctico y uno técnico. En el primero, se encuentra el saber-hacer o la praxis y en el segundo, el saber o logos que justifica ese saber hacer. En el entorno de una institución que privilegia una u otra organización matemática, la actividad escolar se constituye en un tipo de tarea $(T)$ que se abordará con la técnica o conjunto de realizaciones y procedimientos (el saber-hacer) que permiten abordarlas, también en la tecnología $(\theta)$, que serán los objetos matemáticos legitimados para el curso (el saber), como son los teoremas, axiomas y definiciones, que además justifican y hacen inteligible a la técnica, y la teoría $(\Theta)$ que a su vez justifica a la tecnología. La tecnología se refiere no sólo al estudio de las técnicas que contiene, sino a la manera en que de estas últimas se desprenden. En el trabajo reportado, el trabajo se centra la descripción, principalmente en la tecnología (SÁNCHEZ y CAMACHO, 2017).

\section{PRÁCTICAS SOCIALES DESDE LA SOCIOEPISTEMOLOGÍA.}

En este marco teórico se asume una naturaleza de construcción social para el conocimiento matemático y una centralidad de la actividad humana en esa construcción (CARRASCO, DÍAZ Y BUENDÍA, 2014). Esto implica reconocer aquellas prácticas que se ejercen en diversas áreas de actividad humana, y en la cual las ideas matemáticas, conocidas como herramientas, son necesarias y les dan significados. Entonces la acción central en la mirada es entender las prácticas sociales toda vez que son ejercidas por el sujeto social, que están a la base de la actividad. Al decir de Buendía y Montiel (2013) es establecer una epistemología de prácticas en torno a la noción matemática que se quiere enseñar. 


\section{METODOLOGIA}

\section{METODOLOGÍA DE TRABAJO DE LOS DOCENTES}

El trabajo con los profesores se desarrolló en el marco de un primer curso de maestría en educación matemática.

Se consideró el análisis de los relatos de 3 grupos de profesores, en sus vivencias en aula con los estudiantes. Los docentes formaron 3 grupos de tres a cuatro docentes, un integrante de cada grupo eligió un relato de la situación crítica personal de uno de ellos, la que consideraron la más significativa. Estas situaciones se refirieron a preguntas que les hicieron sus estudiantes y que recordaron que no lograron resolver de modo adecuado.

Para el desarrollo del pensamiento crítico de los docentes en formación de la muestra en estudio, se consideraron tres momentos: en el primer momento se aplicó a sus experiencias de aula el relato protagónico; en el segundo momento se aplicó tres teorías para el análisis de lo sucedido: la teoría de registros de representación semiótica, la teoría antropológica de lo didáctico y la teoría socioepistemológica y en el tercer momento se estableció una propuesta didáctica alternativa para la enseñanza del tema enseñado.

En el primer momento: el relato protagónico se estructuró a partir de tres facetas descriptivas integradas: (a) la descripción externo, de la situación desde la posición de un observador objetivo; (b) la descripción del devenir emocional del protagonista que concurrió en la situación vivida y (c) un relato de la racionalidad pedagógica que estuvo a la base de las decisiones didácticas tomadas en la situación. A partir de ello, se analiza, desde diferentes teorías, la situación y la elaboración alternativas de acciones didácticas a su actuar en la situación. A partir de esto, en un segundo momento, los docentes analizaron las facetas descriptivas aplicando los marcos teóricos mencionados anteriormente para abordar el fenómeno didáctico. Para ello se establecieron los siguientes momentos: (a) Selección de un fenómeno didáctico. (b) Análisis desde la Teoría de registros de representación semiótica (DUVAL, 2004), con el propósito de evidenciar el trabajo con las representaciones que usan; (c) Análisis desde la Teoría Antropológica de lo Didáctico (CHEVALLARD, 2007), con el propósito de evidenciar la ac- tividad matemática que está involucrada en la temática y; (d) Análisis desde la teoría Socioepistemológica, cuya intencionalidad es reconocer que la idea enseñada es parte de prácticas profesionales o matemática que les dan sentido y necesidad.

\section{METODOLOGÍA DE INVESTIGACIÓN}

La metodología es un estudio de caso, en el marco de investigación protagónica. El análisis de datos se basó en el análisis conceptual de las descripciones de los equipos de docentes en formación.

\section{RESULTADOS}

Para cada situación vivida con los alumnos, que eligieron los grupos de docentes, éstos declaran que los estudiantes tenían un buen desempeño, o que el contenido involucrado ya lo habían aprendido. Por ello la respuesta no era esperada y surgía la frustración en los docentes.

Los episodios refieren a saberes dados por los profesores y por tanto la respuesta dada, se orienta a recordar lo correcto. Sin embargo en la representación que construye el docente, emerge la duda respecto de la pregunta. El "¿por qué?" de la regla a aplicar y la representación docente que la explicación no logra dar sentido al estudiante.

En este artículo se mostrará el análisis protagónico hecho por el grupo 2, integrado por cuatro docentes.

Primer momento: análisis protagónico

a) La descripción externa de la situación desde la posición de un observador objetivo La docente describe la situación vivida en clase en relación a la pregunta de uno de sus alumnos.

En clase de séptimo grado de educación básica, un alumno preguntó: "Profesora, si tengo menos con menos habiendo una sustracción entre medio, ¿tengo que sumar?" La profesora señala que se da cuenta que su respuesta "no estaba respondiendo a su pregunta, porque logró constatar que el alumno logra comprender el procedimiento que debía realizar, pero no lograba entender la explicación del porqué".

La profesora para dar término a la pregunta del alumno, le dice que en la siguiente clase 
va a resolver el ejercicio y que va a explicar el contenido nuevamente.

Ante la insistencia del alumno que decía que no entendía, varios alumnos se animaron a decir que tampoco entendían. La docente les indicó que desarrollen lo que puedan de la guía, es decir, lo que entiendan y lo que no entiendan lo consideren para preguntar en la siguiente clase. Los alumnos entregaron a la profesora los ejercicios que pudieron resolver y finalizó la clase.

b) La descripción del devenir emocional del protagonista que concurrió en la situación vivida

A continuación, se describe brevemente, la parte emocional que sintió la docente, protagonista de la situación, que es consensuada por todos los docentes integrantes del grupo-

La docente se sintió nerviosa con la pregunta del alumno, a medida que el alumno seguía insistiendo en que no entendía la respuesta que se le había dado. Al evidenciar que el resto de los alumnos también prestan atención a la insistencia del alumno en su pregunta, la profesora aumenta su nerviosismo. Luego, logra regular su emoción y ofrece a los alumnos darles una explicación la siguiente clase.

c) Un relato de la racionalidad pedagógica, en el que se tomaron decisiones didácticas.

La respuesta que le dio la docente al alumno fue explicando la regla de los signos donde le dice al alumno que si tiene dos signos negativos juntos, tiene que transformar esos dos menos en un más.

Los docentes mencionan que la docente usó nemotecnia sin evocar la epistemología de la propiedad.

La explicación que escoge la docente es en base a los contenidos y habilidades desarrolladas en clases anteriores. Ella se da cuenta que su respuesta no se sustentaba didáctica ni epistemológicamente, ya que estaba generando que el estudiante no comprendiera lo que se le intentaba explicar. $Y$ se dio cuenta que eran varios los alumnos que no comprendían el por qué se debía realizar adición cuando el signo indicaba una operatoria de sustracción en algunos ejercicios de la guía de ejercicios.

Para tomar decisiones didácticas al respecto, los docentes analizaron el cuadernillo de ejercicios del séptimo básico. Notaron que existe solo dos representaciones para abordar el objetivo de aprendizaje: "Restar números enteros". La primera es una representación aritmética y la otra es una representación de la resta a partir de un problema en lenguaje verbal. De acuerdo al texto, se menciona que la sustracción de números enteros se realiza sumando al minuendo el inverso aditivo del sustraendo y en el caso de resolver operaciones combinadas de adición y sustracción de enteros se puede: transformar las sustracciones en adiciones y operar de izquierda a derecha. De acuerdo a esto, los docentes mencionan que tampoco el texto responde a la pregunta del alumno. Los docentes, explícitamente, detectan un obstáculo epistemológico cuando mencionan dificultades de comprensión en la génesis del algoritmo que se aplica para restar números enteros; detectan errores conceptuales al mencionar que la repetida frase: "menos por menos es positivo", no tiene variadas representaciones semióticas sino más bien un consenso matemático. $Y$ mencionan que algunos alumnos no asumen una instrucción sin saber el por qué se debe realizar esa acción.

Segundo momento: análisis aplicando los marcos teóricos: teoría de los registros de representación semiótica de Duval, la Teoría de Situaciones didácticas y la socioepistemología.

En cada aspecto, los profesores revisitan la situación problemática y analizan los aspectos que cada teoría ofrece a la situación. Lo que se aprecia es que se centran en mirar más que su actuación los elementos que concurren a la actividad matemática que implica la situación reseñada, develando una complejidad 
de la actividad matemática y contraste con lo simple de su explicación.

Los docentes revisan los textos y guías de trabajo que se usan en la escuela y con las cuales se trabajó en las clases previas.

En un libro de texto, que se da a los alumnos de séptimo grado, analizaron de acuerdo a la teoría antropológica de lo didáctico:

Tipo de Tarea:

Operar con número enteros con paréntesis.

\section{Técnica}

- "Si delante del paréntesis hay un signo más $(+)$, el signo interior se conserva" $+(+3)=+3=3$

$$
+(-2)=-2
$$

- Sí delante del paréntesis hay un signo menos (-), el signo interior se cambia al signo contrario.

$$
\begin{aligned}
& -(+4)=-4 \\
& -(-5)=+5
\end{aligned}
$$

\section{Tecnología}

No se observan instancias donde el estudiante pueda desarrollar su tecnología.

En su informe, muestran cómo la praxeología que prima en el material usado en la enseñanza no aborda los momentos de trabajo de la tecnología y de este modo se evidencia que la duda del estudiante no ha sido por tanto abordada previamente.

Tercer momento: se estableció una propuesta didáctica alternativa para la enseñanza del tema enseñado.

Desde la perspectiva socioepistemológica, los profesores se abocan a entender que actividad humana emerge el uso de aritmética de números negativos y en especial la operación base de la situación crítica; es decir, restar un número negativo. Así, a partir de la experiencia y estudios previos en la que un profesor explora elementos de contabilidad, desde la socioepistemología, se señala "Consideramos que nuestra problemática, "menos por menos es más" es una situación que se da de manera muy recurrente, sobre todo en aquellas prácticas de referencia en donde se debe llevar una contabilización de dinero".

Orientándose de este modo la búsqueda de las acciones contables que dan sentido y necesidad a la operatoria con números negativos comienzan a resignificar el signo positivo y el deber como signo negativo, destacamos el siguiente diálogo:

Prof. 1: "Sii debo $\$ 6000$ pesos, y me quitan la deuda, ya no debo los $\$ 6000$, pesos, eso es menos la deuda, entonces menos, los menos \$6000".

Inv. : "Pero si debo $\$ 6000$ y ya no los debo, no aparecen en mi bolsillo $+\$ 6000 "$

Aquí se muestra como el "deber", se significa como número negativo, y por tanto la situación (-6000) se interpreta como condonar una deuda. Sin embargo la resolución, que implica $-(-6000)=+6000$, se interpreta como si se incrementara lo que tengo en el bolsillo. La significación natural que emerge en el grupo no es coherente con la experiencia cotidiana.

Esto evidencia la dificultad en encontrar contextos reales. El contexto mencionado referido a contabilidad, que si bien ofrece un contexto para el trabajo con negativos, no es de directa significación. Si bien no se construye una descripción de la práctica de contabilidad, dado el tiempo de trabajo, si se aborda una descripción de la actividad contable que pueda dar sentido al menos con menos.

En su análisis se recurre a la ecuación patrimonial (Scarano, 2006) que modela el equilibrio que debiera existir entre los activos, el pasivo y el patrimonio de un sujeto comercial, que se expresa por la igualdad:

$$
\text { Activos }=\text { Pasivos }+ \text { Patrimonio }
$$

y por tanto el patrimonio queda determinado por:

$$
\text { Patrimonio }=\text { Activos }- \text { PAsivos }
$$

Así, el patrimonio, que se diferencia del dinero disponible, es aquello que se posee después de pagar las deudas y obligaciones (pasivos). De este modo, si 
se significa el positivo como aquello que se tiene patrimonialmente, y el negativo como las deudas que se restan a los activos, la resta "-(-6000)=+6000" se significa como un aumento en el patrimonio, toda vez que se ha restado una deuda.

Esto permite dar un contexto de actividad comercial, donde la resta de un número negativo tiene un sentido claro respecto de su resultado, que es coherente con el significado de + .

En el trabajo final el grupo construye una situación de aula que aborda de modo explícito la significación de la situación menos por menos. A partir de la práctica comercial definida, señalan:

Tales prácticas sociales se pueden observar en la relación de compras a créditos o en plazos de pagos definidos, para los estudiantes un nivel muy cercano es la deuda del almacén que para ellos es el término de "fiar" que es confiar la promesa de pago a posterior, en este caso se da la relación de pagar y deber, haciendo un juego que contempla los números negativos,

La secuencia didáctica por tanto, que busca dar significado y justificación a la resta, se ofrece con una mayor complejidad que la evidenciada en el proceso crítico. En particular establecen las siguientes resignificaciones que están a la base de su situación didáctica:

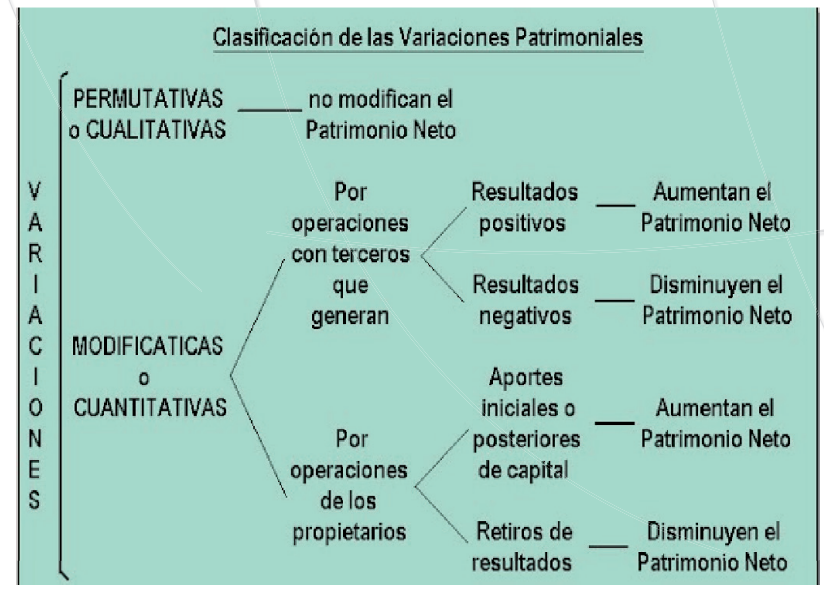

Tomado de http://contabilidad-basica3ero.blogspot. com/2016/10/transacciones-comerciales.html

Finalmente, los demás grupos siguieron un camino similar, con mayor o menor logro en cuanto a las profundizaciones. A continuación se presentan las situaciones elegidas por los otros grupos de docentes:

Grupo 1: "Uno de los alumnos destacados del curso, de buen desempeño y siempre participativo en clases, pregunta: ¿cómo el ángulo va a medir lo mismo que el arco que lo subtiende, si el ángulo se mide en grados y el arco se mide con unidades de medida de longitud?"

Grupo 3: En clase ofrece la siguiente operación aritmética:

$$
\left(-\frac{5}{3}\right) \cdot\left(-\frac{2}{3}\right) \cdot\left(\frac{1}{3}\right)
$$

El profesor pregunta ¿Qué tienen en común estas tres fracciones?, y los estudiantes responden, "el exponente", luego la pregunta del profesor a la clase es: "¿Qué hacemos ahora?" y la respuesta de un estudiantes fue "Igualamos los denominadores". La profesora menciona que realiza inflexión de la voz, aplica voz enérgica, modifica su postura corporal cuestionando la respuesta del estudiante y evidencia cierto grado de frustración y desmotivación, ya que el contenido fue trabajado en el primer módulo y cierra el diálogo con "¿En qué operación igualamos los denominadores?" - Esto es entendido de modo frustrado, pues la suma de fracciones había tenido buenos desempeños en la evaluación.

\section{CONCLUSIONES}

El relato protagónico, de situaciones de los docentes con los alumnos, permitió dar significatividad a los elementos teóricos de las diferentes teorías abordadas. La construcción inicial del relato, que abordó lo emocional y las representaciones docentes, fortaleció una descripción pedagógica de la situación crítica. El relato protagónico se muestra como una interesante propuesta para generar un tránsito del relato en primera persona de lo vivido en el aula, pasando por el relato en segunda persona hacia el relato científico y profesional en tercera persona (Varela, 2000). Así, los grupos de profesores se apropiaron del desafío de innovar una situación crítica vivida, situación cuyo relato los profesores asumen como propio y al cual siempre 
se puede volver para mejorar, toda vez que el protagonista es parte de un grupo formado de profesores.

El trabajo subsecuente, permitió que los profesores, integrantes de los grupos, complejicen la comprensión de aquello que se enseña. En el caso del grupo analizado, los docentes significaron la resta de números negativos aplicando la teoría de registros de representación semiótica y los aspectos de la actividad matemática desde la TAD y también buscaron los contextos de uso de la aritmética de los números negativos. Así la significación de los registros de representación semiótica y, principalmente, el uso restrictivo hecho en la situación crítica, permitió una primera resignificación de lo negativo en la aritmética. El análisis con la teoría TAD posibilitó, al grupo de docentes, reconocer que no se habia abordado en la enseñanza la justificación de la resta de negativos, es decir el trabajo con tecnología estaba ausente del trabajo con estudiantes. El relato, y las profundizaciones, respecto de cómo se vivió el proceso de aprendizaje, evidenció al grupo que se centren en el binomio de la praxis. Finalmente, el determinar una práctica que permita entender la regla "menos con menos, da más" llevó una búsqueda que evidenció la complejidad implícita en la frase que representa la resta de un número negativo.
El relato protagónico de la situación crítica permitió iniciar el estudio de las teorías en educación matemática, desde un contexto real de mejora. El equipo de profesores reconoció un episodio de su propia práctica que era necesaria mejorar, pues la práctica didáctica realizada en el aula no permitió el logro del aprendizaje del producto de números negativos.

De este modo el alcance de las herramientas de las teóricas abordadas, se significan, para los docentes, como útiles para la mejora de su práctica. El hecho que los grupos de profesores reconocieran los aspectos no abordados en la práctica propia y la importancia de incorporarlos, a saber, la diversidad de registros de representación, la justificación de las técnicas usadas y las contextualizaciones. Luego es factible suponer aprendizajes significativos de las teorías abordadas.

Consideramos, por tanto, que el ciclo vivido en este curso, en torno a buscar alternativas para la situación crítica, es una propuesta didáctica relevante para la formación continua y la incorporación de las nuevas herramientas que la teoría en educación matemática nos va ofreciendo. 


\section{REFERÊNCIAS}

ASSAÉL, J.; GUZMAN, I. talleres de Educación Democrática (tED). Cómo aprende y cómo enseña el docente: un debate sobre el perfeccionamiento. Santiago: PIIE/ICI, 1992.

BUENDÍA, G.; MONTIEL, G. Acercamiento socioepistemológico a la historia de las funciones trigonométricas. Disponible en: http://www.repositoriodigital.ipn.mx/bitstream/123456789/11361/1/3\%C2\%BAPOA\%202009\%20Gabriela\%20Buendia\%20 (5).pdf. Access in: 2013.

CARRASCO, E.; DÍAZ, L.; BUENDÍA, G. Figuración de lo que varía. Enseñanza de las ciencias, v. 32, n. 3, p. 365-384, 2014.

CHEVALLARD, Y. El análisis de las prácticas docentes en la teoría antropológica de lo didáctico. Recherches en didactique des mathématiques, $v$. 19, n. 2, p. 221-266, 2013.

DISTÉFANO, M. L.; AZNAR, M. A. G.; POCHULU, M. D. Prácticas matemáticas y funciones semióticas en la significación de representaciones simbólicas de la matemática superior. Revista electrónica de investigación en educación en ciencias, v. 11, n. 2, p. 1-15, 2016.

ETELÄPELTO, A.; VÄHÄSANTANEN, K.; HÖKKÄ, P.; PALONIEMI, S. What is agency? Conceptualizing professional agency at work. Educational Research Review, n. 10, p. 45-65, 2013.

HAMMERNESS, K. To seek, to strive, to find, and not to yield: a look at current conceptions of vision in education. In: Second international handbook of educational change. Springer, Dordrecht. p. 10331048. 2010.

HERNÁNDEZ, M. E. M.; CATRINAO, J. C. C. Liderazgo pedagógico para reestructurar creencias docentes y mejorar prácticas de aula en contexto mapuche. Revista Electrónica Educare, v. 20, n. 1, p. 22, 2016.
KETELAAR, E.; BEIJAARD, D.; BOSHUIZEN, H. P.; DEN BROK, P. J. Teachers' positioning towards an educational innovation in the light of ownership, sense-making and agency. Teaching and Teacher Education, v. 28, n. 2, p. 273-282, 2012.

LABRA, P. Construcción de conocimiento profesional docente: el caso de la formación en la práctica. Tesis para optar al Grado Académico de Doctor en Educación. Universidad Academia de Humanismo Cristiano, 2011.

LASKY, S. A sociocultural approach to understanding teacher identity, agency and professional vulnerability in a context of secondary school reform. Teaching and teacher education, v. 21, n. 8, p. 899-916, 2005.

LIPPONEN, L.; KUMPULAINEN, K. Acting as accountable authors: Creating interactional spaces for agency work in teacher education. Teaching and teacher education, v. 27, n.5, p. 812-819, 2011.

LÓPEZ, G. (2013). Pensamiento crítico en el aula. Docencia e Investigación, 22, p. 41-60.

LORA, L. La ecuación patrimonial. Disponible en: https://es.slideshare.net/lauramelisita/la-ecuacin -patrimonial. Access in: 2019.

NIETO, A. M.; SAIZ, C. Skills and dispositions of critical thinking: are they sufficient?. Anales de Psicología, v. 27, n. 1, p. 202-209, 2016.

SÁNCHEZ LUJÁN, B. I.; y Camacho Ríos, A. Nuevos objetos y nuevas técnicas para la enseñanza de la matemática, 2017.

SCARANO, E. R. ¿ La contabilidad es ciencia o es científica?. Actualidad Contable FACES, v. 9, n. 12, 2006.

REYES, L.; CORNEJO, R.; ARÉVALO, A.; SÁNCHEZ, R. Ser docente y subjetividad histórica en el Chile actual: discursos, prácticas y resistencias. Polis. Revista Latinoamericana, n. 27, 2010.

VARELA, F. El fenómeno de la vida. Santiago: Dolmen, 2000. 